\title{
Necrotizing fasciitis: A case report and a review of the literature
}

\author{
Giovanni Petracca, Francesco Zappia, Giuseppe Maccarone \\ Dipartimento di Chirurgia Generale, Ospedale G. Jazzolino, Vibo Valentia, Italy
}

\begin{abstract}
Necrotizing Fasciitis (NF) is a soft tissue infection characterized by extensive necrosis of subcutaneous fat, neurovascular structures and fascia. The characteristic of this case was aggression and rapid extension of the disease. A 59-years-old woman was observed in a very serious condition, with fever and a large necrotic area that smelled of the right inguinal-femoral region in extension to the thigh. No history of illness. Obesity. Diagnosis may be delayed due to minimal initial cutaneous cellulite. A delay in diagnosis can lead to the progression of the disease shown by shock, multiple organ failure and, ultimately, death. Computed tomography and Magnetic Resonance Imaging could be useful in cases where the signs are equivocal or the diagnosis is in doubt. A detailed review of the literature is presented and the current treatment modalities are described. In this case, the aggressive surgical debridement, the complete medical management of sepsis and comorbidities and the timely closure of the wound by dermo-epidermal grafting were essential for a positive result. NF is an uncommon life-threatening soft tissue infection, which spreads rapidly and progressively along the deep fascia. The prognosis depends on accurate diagnosis and immediate treatment.
\end{abstract}

\section{INTRODUCTION}

Necrotizing Fasciitis (NF) is an uncommon life-threatening soft tissue infection, which spreads rapidly and progressively along the deep fascia. ${ }^{1}$

Correspondence: Giovanni Petracca, Department General Surgery, "G. Jazzolino" Hospital Vibo Valentia, Piazza Fleming, 89900 Vibo Valentia, Italy.

Tel.: +39.0963.962252-4

E-mail: doc37@virgilio.it

Key words: Necrotizing fasciitis; shock; skin graft; case report.

Conflict of interest: The authors declare no conflict of interest.

Availability of data and materials: All data underlying the findings are fully available.

Ethics approval and consent to participate: No ethical committee approval was required for this case report by the Department, because this article does not contain any studies with human participants or animals. Informed consent was obtained from the patient included in this study.

Consent for publication: The patient gave her written consent to use her personal data for the publication of this case report and any accompanying images.

Received for publication: 19 March 2020.

Accepted for publication: 30 March 2021.

This work is licensed under a Creative Commons Attribution NonCommercial 4.0 License (CC BY-NC 4.0).

${ }^{\circ}$ Copyright: the Author(s), 2021

Licensee PAGEPress, Italy

Italian Journal of Wound Care 2021; 5(2):60

doi:10.4081/ijwc.2021.60
It was named necrotizing fasciitis in 1952, from necrosis, which means death of a portion of tissue, and fascia, which refers to the fibrous tissues that enclose and connect the muscles. ${ }^{2}$

The predisposing conditions are mainly diabetes mellitus, alcoholism, end-stage renal disease, malignancy, liver cirrhosis, malnutrition, gout, corticosteroid use, and trauma. $^{3-6}$

Diagnosis may be delayed because of the initial minimal skin cellulitis, even as the subcutaneous tissue suffers extensive necrosis and pronounced systemic toxicity develops. $^{7}$

The progression of the disease is fulminant and the mortality is high, despite effective antibiotic treatment. ${ }^{8,9}$

The prognosis depends on accurate diagnosis and immediate treatment. In addition to delay in diagnosis and treatment, several risk factors, such as more than one underlying condition, thrombocytopenia, anemia, and older age, have been shown to influence mortality. ${ }^{10}$

Higher mortality in patients with streptococcal toxic shock syndrome and in disease associated with certain bacteria has been reported. ${ }^{11-13}$

NF death rate is very high $(20-40 \%){ }^{14}$

\section{CASE REPORT}

A 59-years-old woman was got into observation in a very serious condition, stupor with fever and extensive necrotic area smelling of the right inguinal-femoral region in extension to the thigh (Figure 1). No history of disease. Obesity. Heart rate being $105 \mathrm{bpm}$, respiratory F of 25 acts/minute and $\mathrm{AP}$ of $80 / 60 \mathrm{mmHg}$.

Laboratory parameters showed only a glycaemia alteration $(338 \mathrm{mg} / 100 \mathrm{~mL})$ and a leucocytosis $(16.000 \mathrm{WBC}$

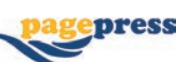


with a neutrophilia equal to $80 \%$ ). Thorax X-ray were normal. ECG was sinus rhythm with rate $105 \mathrm{bpm}$. CT scan shows gas bubbles in the subcutaneous tissue in the groin and thigh.

It was then started a wide-spectrum antibiotics therapy(Meropenem 1g x 3/die; Teicoplanina $400 \mathrm{mg}$ x 2/die), antifungal therapy (Flucanazolo $400 \mathrm{mg} /$ die), rehydration therapy, Low-Molecular-Weight Heparin (LMWH).The patient was subjected, under general anesthesia, a debridement until the appearance of vital tissue. Multiple biopsies carried out during the operation too underwent cultural and histomorphopathological analysis. After just 24 hours there took place a rapid development of the clinical picture with the appearance of areas of cutaneous necrosis extending to the right knee.

Every day it was carried debridement until the gradual achievement of the fascia (two weeks after admission, Figure 2).

Gradually, the general conditions have improved. Given a good toilet of the wound, he began, after about two weeks of admission, treatment with hyperbaric oxygen therapy for eight cycles. After about a month, the wound was clean with granulation tissue formation (Figure 3).

After about two months of daily medication, there was a significant reduction of the wound surface.

She then underwent surgery of wound reconstruction, by partial-thickness skin graft (Figure 4).

After seven days, the skin graft area was healed (Figure 2) and after ten days, the skin donor site was healed (Figure 5).

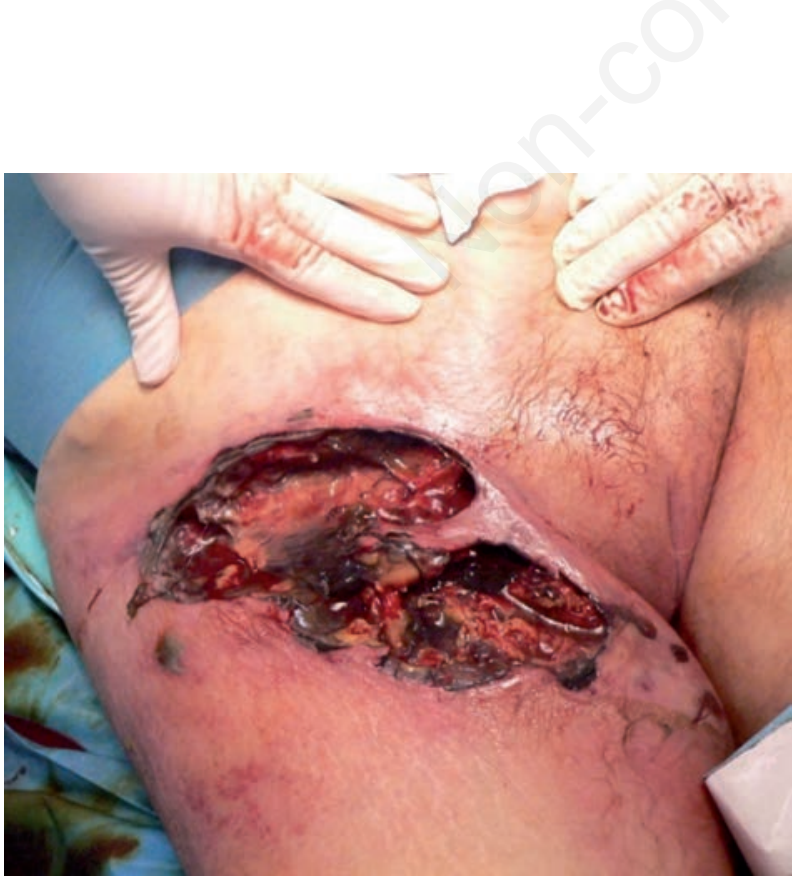

Figure 1. Necrotic area smelling of the right inguinal-femoral region.

\section{DISCUSSION}

Necrotizing skin infections were first described by Jones in 1871, although at the time the term hospital gangrene was used. ${ }^{15}$

The term necrotizing fasciitis was coined by Wilson in the 1950s to describe necrosis of the fascia and subcutaneous tissue with relative sparing of the underlying muscle. ${ }^{2}$

NF is characterized by rapid destruction of tissue, systemic toxicity, and, if not treated aggressively, gross morbidity and mortality. Early diagnosis and aggressive surgical treatment reduces risk; however, it is often difficult to diagnose NF, and sometimes patients are treated for simple cellulitis until they rapidly deteriorate. ${ }^{16}$

Antibiotic therapy is mandatory, and early surgical exploration and debridement is critical to ensuring a good outcome.

Different terms and classifications have been used to describe necrotizing infections of the skin and subcutaneous tissue. These include necrotizing fasciitis, synergis-

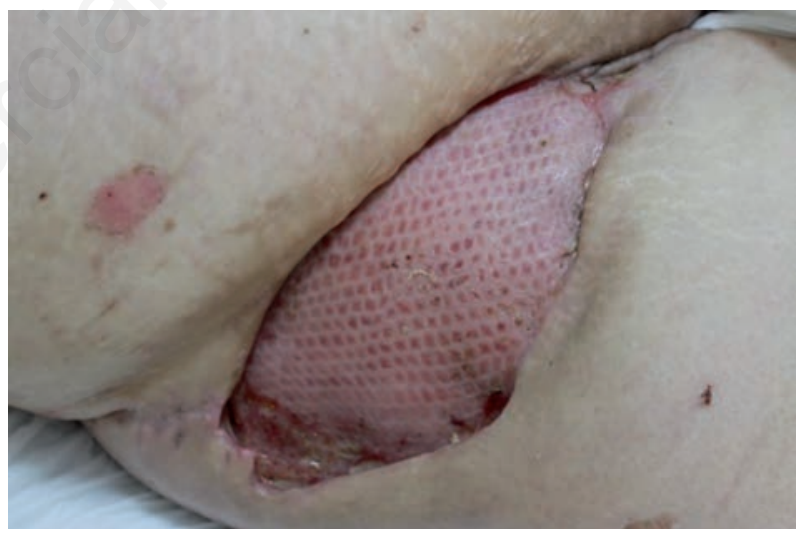

Figure 2. Area skin graft after seven days.

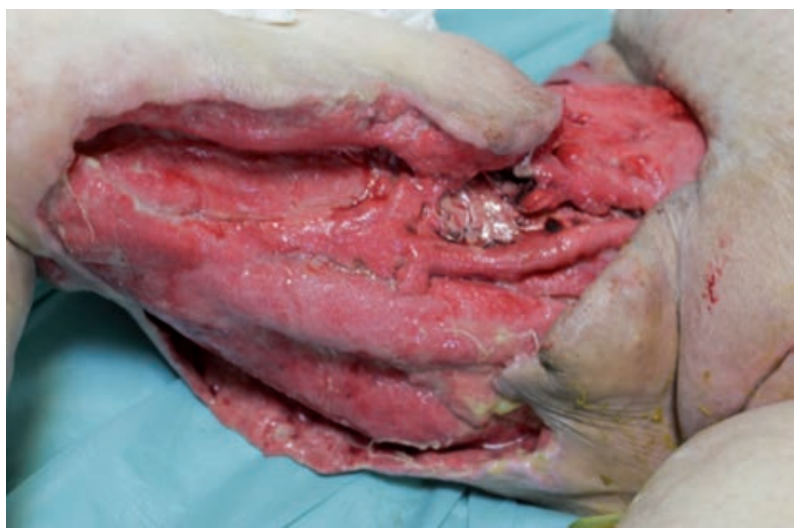

Figure 3. After about a month, granulation tissue formation. 
tic necrotizing cellulitis, streptococcal myonecrosis, and gas gangrene. This variety of classifications and terminology has been based on affected anatomy, microbial cause, and depth of infection.

FN can be classified in two types: i) Type 1 or polymicrobial: caused by aerobic and anaerobic bacteria, it target patients showing one or more risk factors (subjects aged50 or over, diabetes mellitus, immunodeficiency, past surgical operation, penetrating trauma, omphalitis in newborns, colon carcinoma pierced colichdiverticola, appendicular abscess etc.) it is the most frequent type (about $80 \%$ of the cases); ii) Type 2 or monomicrobial: it's caused by group A Streptococci only (or in association whit the Staphylococcus aureus), it targets subjects of any age who do not present any risk factor; it makes up for the $20 \%$ of the cases. ${ }^{14}$

\section{Pathology}

Most cases commence with trauma to the skin surface (e.g., from a penetrative injury), with seeding of bacteria. Infection starts in the deep tissue planes, and the epider-

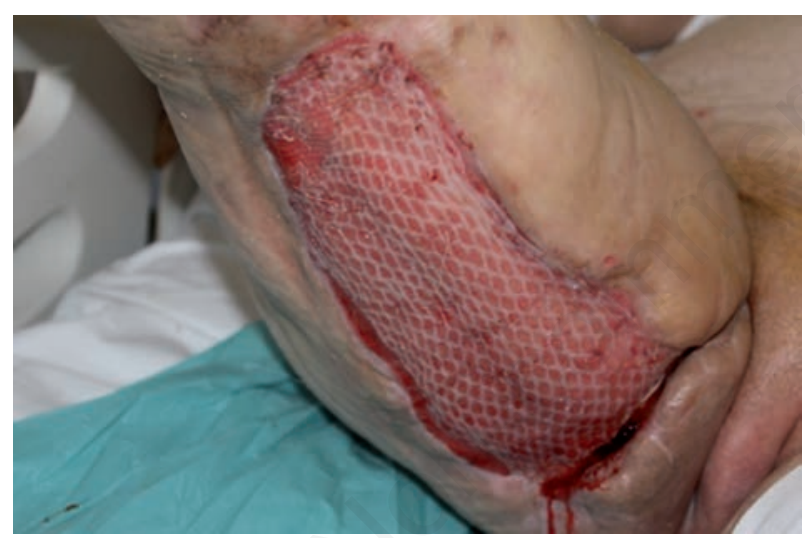

Figure 4. Partial-thickness skin graft.

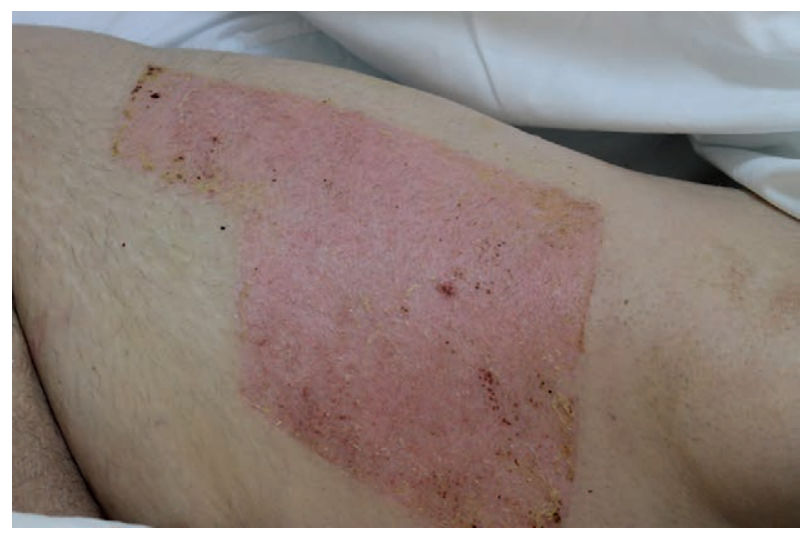

Figure 5. Area skin graft after seven days. mis might not be initially affected. The clinical disease is expressed when infective organisms spread through the tissue along the deep fascia. Bacteria rapidly multiply within viable tissue, although fibrous attachments between subcutaneous tissues and fasciae limit spread to areas like the hands, feet, and scalp. Infection also spreads to venous and lymphatic channels, leading to edema. The spread of bacteria results in thrombosis of blood vessels in dermal papilla, resulting in ischemia and gangrene of subcutaneous fat and dermis. ${ }^{17}$ If the fascia is breached, infection of the muscle leading to myositis occurs. Gasproducing organisms such as Clostridium species can give rise to subcutaneous gas, from which stems the descriptive term gas gangrene.

In addition, infections with toxin-producing bacteria (S. Aureus and S. Pyogenes) can lead to a toxic shocklike syndrome. Therefore, seemingly limited infection can result in septic shock and multiorgan failure.

\section{Risk factors}

Often, patients presenting with NF have some predisposition to infection (Diabetes, Chronic disease, immunosuppressive drugs (e.g. prednisolone), malnutrition, age $>60$ years, intravenous drug misuse, peripheral vascular disease, renal failure, underlying malignancy, obesity).

Precipitating events causing NF: i) Traumatic: Surgery, minor invasive procedures (e.g. joint aspirations, acupuncture), intravenous drug use, penetrating injuries (e.g. insect and animal bites); ii) Nontraumatic: Soft tissue infection, burns, childbirth. ${ }^{18}$

Risk factors for NF in the pediatric population include malnutrition and skin infections such as varicella. ${ }^{19,20}$

\section{Clinical features}

Patients with NF can present with constitutional symptoms of sepsis (e.g. fever, tachycardia, altered mental state, diabetic ketoacidosis) alone or with evidence of skin inflammation, which makes diagnos is a little more straightforward.

Limbs are among the most common sites of infection. Common sites of infection included the lower extremities (28\%), upper extremities (27\%), perineum (21\%), trunk $(18 \%)$, and the head and neck (5\%). ${ }^{21}$

Patients with NF are usually systemically toxic, initially presenting with fever (temperature higher than $38^{\circ} \mathrm{C}$ ), tachycardia, diaphoresis, and possibly even an altered mental state or diabetic ketoacidosis. The physical examination should include all parts of the body to search for skin inflammation. This is especially necessary for patients who present with sepsis of which the source is not obvious. ${ }^{22}$

Most patients present with signs of skin inflammation (i.e., pain, skin edema, and erythema). 
NF typically presents with patchy discoloration of the skin with pain and swelling, but without a defined margin or lymphangitis. ${ }^{23}$

Progression of NF is marked with the development of tense edema, a grayish-brown discharge, vesicles, bullae, necrosis, and crepitus. Hemorrhagic bullae and crepitus are sinister signs, with the likelihood of underlying fascia and muscle being compromised. ${ }^{24}$

Localized pain is another clue to NF. As the disease is a deep-seated infection, the epidermis is minimally involved at initial presentation. The patient might complain of pain out of proportion to the degree of dermal involvement or pain that extends past the apparent margin of infection. Certain patients, notably those with diabetic neuropathy with loss of sensation, can experience minimal pain, resulting in a missed diagnosis. This is especially likely in concealed sites of infection, such as the perineum or oral cavity. A patch of anesthesia over the site of erythema is

also sometimes described in NF. This is thought to be due to infarction of cutaneous nerves in necrotic subcutaneous fascia and soft tissue. ${ }^{25}$

\section{Disease progression}

NF can follow a hyperacute or a subacute course of progression. The patient with a hyperacute course presents with sepsis and rapidly progresses to multiorgan failure. Diagnosis of sepsis is obvious, and these patients are hospitalized. Several authors have described a subacute variation of NF. ${ }^{26-28}$

These patients have an indolent disease course, with festering soft tissue infection. After the infection reaches a certain threshold, sudden deterioration is an important clinical feature. Aggressive surgical debridement is the cornerstone of treatment in these cases. Progression of disease is invariable in this group, and a delay in diagnosis can lead to greater soft tissue loss and mortality.

\section{Diagnosis and decision for surgical exploration}

The criterion standard for diagnosis of NF is histology of tissue specimens obtained during surgical exploration, which was demonstrated in our case description. During surgical exploration, tissue integrity and depth of invasion can be assessed. Fascial necrosis and loss of fascial integrity indicate a necrotizing infection. Muscle involvement and necrosis are indicative of an advanced stage.

To help decide which patients require surgical exploration, particularly in those with equivocal clinical signs, laboratory and radiologic tests might sometimes be useful.

Leukocytosis with neutrophilia, acidosis, altered coagulation profile, impaired renal function, raised creatinine kinase levels, and raised inflammatory markers, such as C-reactive protein levels, are all helpful if viewed within the whole of the clinical context.

Plain X-ray films can demonstrate subcutaneous gas, but this is a specific not a sensitive finding (positive in fewer than $25 \%$ of cases) and absence of gas does not exclude NF. ${ }^{29}$

Computed tomography and Magnetic Resonance Imaging (MRI) might be useful in cases where signs are equivocal or diagnosis is in doubt. Asymmetrical fascial thickening, fat stranding, and gas tracking along fascial planes are important imaging findings. Computed tomography scans are estimated to have a sensitivity of $80 \%$ for detecting necrotizing soft tissue infections. ${ }^{30}$

In summary, if clinical suspicion is high, surgeons can opt to explore and perform tissue biopsies rather than delay treatment for imaging studies. Additional bedside tests include needle aspiration

and incision biopsy. Negative results, however, cannot exclude NF. Surgical exploration is preferable. Macroscopic findings during surgical exploration include gray necrotic tissue, lack of bleeding, thrombosed vessels, "dishwater" pus, non-contracting muscle, and a positive "finger test" result, which is characterized by lack of resistance to finger dissection in normally adherent tissues.

\section{Treatment}

Once the diagnosis is made, treatment must begin on multiple fronts. First, surgical consultations should be urgently requested with the intention of early wound debridement for collection of tissue cultures, excision of all nonviable tissue, and delineation of the extent of the disease (this is also important as tissue hypoxia limits the efficacy of intravenous antibiotics). It is important to educate patients about the gravity of their condition and the risk of increased mortality if surgical debridement is not performed.

Until blood culture results are available, wide spectrum coverage with intravenous antibiotics is started. These antibiotics cover S. Pyogenes, S. Aureus (including community-acquired MRSA if indicated, according to local resistance patterns), and Gram-negative aerobes and anaerobes as clinically indicated.

Surgical treatment involves a large removal of all the necrotizing tissue through skin and subcutaneous incisions as far as the fascia, in parallel to the vessels and the nerves that have to be isolated and not touched. The adipose tissue and the fascia have to be excised. The surgical openings are left open for subsequent medications carried out every 24 hours or less in the operating room, and possibly for a surgical "second-look". 14

During the operation exudate samples and tissue biopsies will be collected and relative culural and cytological test will be carried out.

Hyperbaric oxygen has also been used as an adjunct to surgery and antibiotics. Its role is still ill-defined. Some 
authors have reported a reduction in mortality, morbidity, and need for repeated debridement in up to two-thirds of cases. ${ }^{31,32}$

Well-controlled randomized controlled trials are still lacking.

Moreover, a retrospective review by Golger et al. showed that morbidity associated with NF was higher in patients who underwent hyperbaric oxygen therapy. ${ }^{21}$

\section{Prognosis}

Mortality due to NF is considerable. Without surgical intervention, mortality approaches $100 \%$. More recent data indicate a mortality of $16.4 \%$ for community-acquired necrotizing

soft tissue infections and $36.3 \%$ for post-procedural necrotizing infections. All of these patients were managed in hospital with intravenous antibiotics and surgical interventions. Considering medical progress in the last 135 years, mortality is still substantial. Diabetic patients, especially those presenting with diabetic ketoacidosis or hyperosmolar hyperglycemic non ketotic acidosis have higher rates of death and longer lengths of hospital stay. ${ }^{33}$

A delay in surgery of more than 24 hours was an independent risk factor for mortality. ${ }^{1}$

\section{CONCLUSIONS}

NF is an uncommon life-threatening soft tissue infection, which spreads rapidly and progressively along the deep fascia. The predisposing conditions are mainly diabetes mellitus, alcoholism, end-stage renal disease, malignancy, liver cirrhosis, malnutrition, gout, corticosteroid use, and trauma. The progression of the disease is fulminant and the mortality is high, despite effective antibiotic treatment. That is why early diagnosis is needed.

Wide-spectrum antibiotic therapy followed up by surgery and, when it is possible, by hyperbaric therapy as well, thus aiming at a substantial reduction of both morbidity and mortality rates, unfortunately still very high.

The prognosis depends on accurate diagnosis and immediate treatment. In addition to delay in diagnosis and treatment, several risk factors, such as more than one underlying condition, thrombocytopenia, anemia, and older age, have been shown to influence mortality.

\section{REFERENCES}

1. Wong $\mathrm{CH}$, Chang HC, Pasupathy S, et al. Necrotizing fasciitis: clinical presentation, microbiology, and determinants of mortality. J Bone Joint Surg Am 2003;85:1454-60.

2. Wilson B. Necrotizing fasciitis. Am Surg 1952;18:416-31.

3. McHenry CR, Piotrowski JJ, Petrinic D, Malangoni MA. Determinants of mortality for necrotizing soft-tissue infections. Ann Surg 1995;221:558-63.
4. Elliott DC, Kufera JA, Myers RA. Necrotizing soft tissue infections. Risk factors for mortality and strategies for management. Ann Surg 1996;224:672-83.

5. Cheng NC, Tai HC, Tang YB, et al. Necrotising fasciitis: clinical features in patients with liver cirrhosis. Br J Plast Surg 2005;58:702-7.

6. Yu KH, Ho HH, Chen JY, Luo SF. Gout complicated with necrotizing fasciitis - report of 15 cases. Rheumatology (Oxford) 2004;43:518-21.

7. Swartz MN. Cellulitis and subcutaneous tissue infections. In: Mandell GL, Bennett JE, Dolin R, eds. Principles and practice of infectious diseases. 6th ed. PhIladelphia: Elsevier Churchill Livingstone; 2005: p. 1172-94.

8. Childers BJ, Potyondy LD, Nachreiner R, et al. Necrotizing fasciitis: a fourteen-year retrospective study of 163 consecutive patients. Am Surg 2002;68:109-16.

9. Taviloglu K, Cabioglu N, Cagatay A, et al. Idiopathic necrotizing fasciitis: risk factors and strategies for management. Am Surg 2005;71:315-20.

10. Liu YM, Chi CY, Ho MW, et al. Microbiology and factors affecting mortality in necrotizing fasciitis. J Microbiol Immunol Infect 2005;38:430-5.

11. Golger A, Ching S, Goldsmith $\mathrm{CH}$, et al. Mortality in patients with necrotizing fasciitis. Plast Reconstr Surg 2007;119:1803-7.

12. Meisel M, Schultz-Coulon HJ. Lebensbedrohliche nekrotisierende Fasciitis colli durch Serratia marcescens [Lifethreatening necrotizing fasciitis colli caused by Serratia marcescens]. HNO 2009;57:1071-4.

13. Tsai YH, Hsu RW, Huang TJ, et al. Necrotizing soft-tissue infections and sepsis caused by Vibrio vulnificus compared with those caused by Aeromonas species. J Bone Joint Surg Am 2007;89:631-6.

14. Costanzo M, Caruso LAM, Condorelli F, et al. Necroting fasciitis case report. Ann Ital Chir 2008;79:299-302.

15. Jones J. Investigation upon the nature, causes and treatment of hospital gangrene as prevailed in the Confederate armies 1861-1865. In: United States Sanitary Commission, editor. Surgical memoirs of the War of the Rebellion. New York, NY: Hurd and Houghton; 1871: p. 142-580.

16. Wang YS, Wong CH, Tay YK. Staging of necrotising fasciitis based on the evolving cutaneous features. Int J Dermatol2007;46:1036-41.

17. Seal DV. Necrotizing fasciitis. Curr Opin Infect Dis2001;14: 127-32.

18. Puvanendran R, Chan J, Huey M, Pasupathy S. Necrotizing fasciitis. Can Fam Physician 2009; 55: 981-7.

19. Fustes-Morales A, Gutierrez-Castrellon P, Duran-Mckinster C, et al. Necrotizing fasciitis: report of 39 paediatric cases. Arch Dermatol2002;138:893-9.

20. Brogan TV, Nizet V, Waldhausen JH, et akl. Group A streptococcal necrotizing fasciitis complicating primary varicella: a series of fourteen patients. Pediatr Infect Dis J1995;14:588-94.

21. Golger A, Ching S, Goldsmith $\mathrm{CH}$, et al. Mortality in patients with necrotizing fasciitis. Plast Reconstr Surg2007; 119:1803-7.

22. Hill MK, Sanders CV. Necrotizing and gangrenous soft tissue infections. In: Sanders CV, Nesbitt LT Jr, editors. The skin and infection: a color atlas and text. Baltimore, MD: 
Lipincott, Williams \& Wilkins; 1995: p. 62-75.

23. Green RJ, Dafoe DC, Raffi n TA. Necrotizing fasciitis. Chest1996;110:219-29.

24. Hsiao CT, Lin LJ, Shiao CJ, et al. Hemorrhagic bullae are not only skin deep. Am J Emerg Med2008;26:316-9.

25. Dufel S, Martino M. Simple cellulitis or a more serious infection?J Fam Pract2006;55:396-400.

26. Wong $\mathrm{CH}$, Wang YS. The diagnosis of necrotizing fasciitis. Curr Opin Infect Dis 2005;18:101-6.

27. Wong $\mathrm{CH}$, Wang TS. What is subacute necrotising fasciitis? A proposed clinical diagnostic criteria. J Infect 2006;52:415-9.

28. Jarrett P, Rademaker M, Duffi 11 M. The clinical spectrum of necrotising fasciitis. A review of 15 cases. Aust N Z J Med1997;27:29-34.
29. Lim YJ, Yong FC, Wong CH, Tan AB. Necrotising fasciitis and traditional medical therapy - a dangerous liaison. Ann Acad Med Singapore2006;35:270-3.

30. Wyoski MG, Santora TA, Shah RM, Friedman AC. Necrotizing fasciitis: CT characteristics. Radiology1997;203:859-63.

31. Riseman JA, Zamboni WA, Curtis A, et al. Hyperbaric oxygen therapy for necrotizing fasciitis reduces mortality and the need for debridements. Surgery 1990;108:847-50.

32. Jallali N, Withey S, Butler PE. Hyperbaric oxygen as adjuvant therapy in the management of necrotizing fasciitis. Am J Surg2005;189:462-6.

33. Health Protection Agency, Group A Streptococcus Working Group. Interim UK guidelines for management of close community contacts of invasive group A streptococcal disease. Commun Dis Public Health2004;7:354-61. 